\title{
An update on the School of Medicine's Medical Student Research Program at Texas Tech University Health Sciences Center
}

\author{
Gurvinder Kaur PhD, Jannette M. Dufour PhD
}

Medical students have been involved in making ground-breaking medical discoveries. The discovery of heparin, insulin, Fogarty catheters, ether anesthesia, spermatozoa, and Raynaud's phenomenon are all examples of scientific discoveries made by medical students. ${ }^{1}$ Banting and Best are known for their discovery of insulin. Frederick Grant Banting, an orthopedic surgeon, believed that the "active principle" of the pancreas was probably destroyed by digestive enzymes in the acinar tissue of the pancreas during extraction of the whole pancreas. ${ }^{2}$ Thus, he hypothesized that ligating the pancreatic ducts of dogs would result in degeneration of the acinar tissue, thereby leaving healthy islets and that isolating the internal secretion(s) from these islets would relieve glycosuria. Charles Herbert Best, a student, worked with Banting on this research project over the summer of 1921. They first tested their pancreatic extract in a diabetic dog and demonstrated that injection of this extract reduced hyperglycemia and glycosuria. In February 1922, Banting and Best treated their first patient suffering from diabetes, 14-year old Leonard Thompson. In 1923, Banting was awarded the Nobel Prize in physiology and medicine for the discovery of insulin. Later, Banting shared his prize money with Best and publicly acknowledged Best's contribution towards the discovery of insulin. ${ }^{2}$ Thus, in this scenario a summer research opportunity for a student led to an important discovery.

Engagement of medical students in research teaches them to ask the right questions, formulate hypotheses, critically review the scientific literature, and document their work in a coherent and accurate

Corresponding author: Gurvinder Kaur Contact Information: Gurvinder.kaur@ttuhsc.edu DOI: $10.12746 /$ swrccc.v6i24.471 manner. These skills, which are tied to research exposure, may help instill a culture of evidence-based medicine among physicians. Amgad, et al. performed an integrated mixed-methods systemic review and meta-analysis of published studies on medical students' participation in research. ${ }^{3}$ They demonstrated that medical student participation in research is associated with improved scientific productivity (both shortand long-term), more informed career choices, and improved knowledge and interest toward research. ${ }^{3}$ Additionally, a demonstrated research experience during medical school is becoming increasingly important to enhance competitiveness of medical students for residencies. In 2016, the National Resident Matching Program conducted a survey of program directors participating in the Main Residency Match to identify the factors that program directors use to select applicants to interview and rank applicants for the Match. ${ }^{4}$ In this report, questionnaires were sent to 3,599 program directors for all specialties and 1,435 responded. For highly competitive residencies, such as dermatology, neurological surgery, orthopedic surgery, otolaryngology, plastic surgery, radiation oncology, and vascular surgery, the percentage of program directors who consider research when choosing applicants to interview was above $65 \%{ }^{4}$ For neurological surgery and radiation oncology it was $83 \%$ and $85 \%$, respectively. ${ }^{4}$ Even in less competitive residency programs, such as family medicine, around two research experiences and at least two publications, abstracts, or presentations were reported for both students matched and unmatched into residency programs, indicating that at least some research exposure is considered for all fields.

In order to provide an opportunity for medical students to participate in a meaningful research experience, the Texas Tech University Health Sciences Center (TTUHSC) School of Medicine created the Medical Student Summer Research Program 
(MSSRP) in 2010. ${ }^{5}$ This is an eight-week program for first-year medical students that allows them to participate in research with established mentors (basic scientist or clinician) over the summer between years 1 and 2 of medical school. After successful completion of this program, participants are encouraged to present posters of their research results during the TTUHSC Student Research Week (SRW) the following spring. ${ }^{5}$ This program has been very successful and has increased interest in research among medical students as demonstrated by a significant number of medical students performing research and presenting their research findings during SRW at the TTUHSC. ${ }^{5}$ In 2017, 81 students participated in the MSSRP, and 57 students (or $70 \%$ ) presented their research work as a first author or co-author at the SRW 2018. There were a total of 44 mentors, clinical $(n=29)$ and basic science $(n=15)$, who participated in the 2017 MSSRP. This issue of the Southwest Respiratory and Critical Care Chronicles highlights the research performed by medical students during the summer of 2017 and has a sample of abstracts presented by medical students at SRW in the Spring of 2018.

Overall, the creation of the MSSRP has increased the number of medical students participating in research and presenting their research findings at TTUHSC. Based on the American Association of Medical Colleges medical school graduation questionnaire, the average participation in research by medical students had increased from $35.7 \%$ in 2005 to $74.2 \%$ in 2017 . On the same note, the percentage of students who reported an authorship on a peer-reviewed oral or poster presentation was slightly above the national average $(53.8 \%$ versus $53.3 \%$ ) in 2017. In the future, additional measures such as examining the success rate of students who participated in research and residency match of their choice will provide a true measure of the success of this program.

Keywords: research, medical students, education, Texas Tech University Health Sciences Center

Article citation: Kaur G, Dufour JM. An update on the School of Medicine's Medical Student Research Program at Texas Tech University Health Sciences Center. The Southwest Respiratory and Critical Care Chronicles 2018;6(24):1-2.

From: Departments of Cell Biology and Biochemistry (JMD) and Medical Education (GK), Texas Tech University Health Sciences Center in Lubbock, TX

Submitted: 5/2/2018

Conflicts of interest: None

This work is licensed under a Creative Commons Attribution-ShareAlike 4.0 International License.

\section{REFERENCES}

1. Stringer MD, Ahmadi O. Famous discoveries by medical students. ANZ J Surg 2009; 79:901-908.

2. Rosenfeld L. Insulin: discovery and controversy. Clinical Chemistry 2002;48:2270-2288.

3. Amgad M, Man Kin Tsui M, Liptrott SJ, Shash E. Medical student research: an integrated mixed-methods systematic review and meta-analysis. PLoS One 2015;10: e0127470.

4. National Resident Matching Program, Data Release and Research Committee: Results of the 2016 NRMP Program Director Survey. National Resident Matching Program, Washington, DC.

5. Dufour JM, Gregorcyk E, Berk SL. Medical student research at Texas Tech University Health Sciences Center: increasing research participation with a summer research program. Southwest Respiratory and Critical Care Chronicles 2017; 5(18): 3-9. 